\title{
Les pérégrinations de Guy Bernède, acteur et témoin de la coopération télévisuelle post-indépendances
}

\section{De l'Ocora Télévision à FR3 Dom-Tom (1962-1975)}

\author{
Flora Losch
}

Citer cet article : Losch Flora (2021), « Les pérégrinations de Guy Bernède, acteur et témoin de la coopération télévisuelle post-indépendances. De l'Ocora Télévision à FR3 Dom-Tom (1962-1975) », Revue d'Histoire

Contemporaine de l'Afrique, $\mathrm{n}^{\circ} 1,143-166$, en ligne. URL :

https://oap.unige.ch/journals/rhca/article/view/01.losch

Mise en ligne : 7 janvier 2021

DOI : https://doi.org/10.51185/journals/rhca.2021.e294

\section{Résumé}

À la fin des années 1950, le développement de la télévision s'est accéléré alors que la France démantelait son empire et que les États africains membres de la Communauté française s'apprêtaient à accéder à la souveraineté internationale. Du fait de cette contingence historique, les nouvelles télévisions nationales d'Afrique francophone et l'audiovisuel extérieur français ont été entremêlés et impactés ensemble au lendemain des indépendances, en relation à l'ordre géopolitique plus large de la Guerre froide. Cet article propose de suivre Guy Bernède, acteur et témoin de la coopération télévisuelle bilatérale postindépendances, depuis la création de l'Ocora Télévision en 1962 jusqu'à la création de FR3 Dom-Tom en 1975. Cet itinéraire témoigne des recompositions de la coopération télévisuelle dans le temps, ainsi que des circulations multiples entre la France et l'Afrique à cette période dans le domaine audiovisuel.

Mots-clés : Congo ; Inter-TV ; Guy Bernède ; Ocora Télévision ; satellite ; Sénégal

The peregrinations of Guy Bernède, actor and witness of televisual cooperation in the postindependence period. From Ocora Television to FR3 Dom-Tom (1962-1975)

\section{Abstract}

In the late 1950s, as France dismantled its empire and the African member states of the French Community were on the verge of gaining independence, the development of television accelerated. As a result of this historical contingency, the new national televisions in Francophone Africa and the French foreign broadcast sector were intertwined and impacted together in the aftermath of independence, in the context of the wider geopolitical Cold War order. This article seeks to follow Guy Bernède, actor and witness of the bilateral televisual cooperation in the post-independence period, from the creation of Ocora Television in 1962 to that of FR3 Dom-Tom in 1975. His itinerary shows the reconfigurations of televisual cooperation over time, as well as the multiple circulations between France and Africa in the audiovisual field during this period.

Keywords: Congo; Inter-TV; Guy Bernède; Ocora Television; Satellite; Sénégal 
Quand j'étais en Afrique, j'étais Africain. J'étais Congolais, j'étais Gabonais, Malgache. Bon, simplement, s'il y avait une rivalité entre Transtel [la coopération allemande] et nous, j'essayais de faire pencher la balance du côté français quand même. ${ }^{1}$

Au lendemain des indépendances de 1960, Guy Bernède a été responsable de la création des télévisions nationales dans les nouveaux «États africains et malgache » pour le compte de I'Office français de coopération radiophonique (Ocora). Rien n'avait pourtant prédestiné cet homme, passionné de sport et de cinéma et qui enfant rêvait de devenir radioreporter, à une carrière dans la coopération.

Né en 1928 à Paris et formé en autodidacte, Guy Bernède avait débuté sa carrière en 1946 comme assistant-réalisateur dans le cinéma et comme producteur-présentateur à la radio $^{2}$. En 1952, il avait obtenu le poste de rédacteur en chef et réalisateur de l'émission sportive "Le dimanche des auditeurs » à Radio Luxembourg (RL) ${ }^{3}$, où il créa le Service des sports et devint dans le même temps l'un des reporters du Service actualités dirigé par Jean Grandmougin. Recruté par I'Ocora en 1962, il rejoint la coopération télévisuelle, où il reste jusqu'à son départ à la retraite en 1991, à une période où les États africains indépendants cherchent à créer leur télévision nationale, symbole de souveraineté et de développement, et où la France, en concurrence avec d'autres nations, cherche à investir ce nouveau média audiovisuel afin d'assurer sa future présence sur le continent ${ }^{4}$.

Ainsi, le parcours de Guy Bernède se situe au carrefour de multiples histoires, en particulier celle des télévisions d'Afrique et celle de la coopération française avec l'Afrique, dont I'enchâssement reste peu explorés. Avec cet objectif et afin de montrer quelques-unes de leurs facettes, cet article propose de suivre cet itinéraire entre 1962 et 1975, deux dates qui correspondent à des moments-clés de la carrière de Guy Bernède et qui ont aussi été des dates charnières de la coopération télévisuelle avec l'Afrique. En effet, 1962 a été l'année de la création de l'Agence Télévision de l'Ocora, qui marque le début de son parcours de coopérant. 1975 ensuite, a été celle de la création de FR3 Dom-Tom, une restructuration aux effets délétères pour la coopération avec les télévisions d'Afrique francophone et qui ouvre la période la plus difficile de sa carrière.

Dans un premier temps, nous nous intéresserons à la période de l'Ocora Télévision (19621969) à laquelle est associé le démarrage de la Télévision congolaise à Brazzaville, le premier à avoir eu lieu en Afrique subsaharienne francophone en 1962 et le premier auquel Guy Bernède

\footnotetext{
1 Entretien avec Guy Bernède, 21 et 22 novembre 2019, Boulogne-Billancourt. Je tiens à remercier tous les relecteurs pour leurs remarques avisées et leur aide dans la construction de cet article.

2 Durant sa mobilisation en 1948-1949, Guy Bernède avait également été affecté au Service cinématographique de I'Armée (SCA), dans le premier contingent du Fort d'Ivry.

3 Ancienne radio associative créée à Luxembourg en 1925, RL (aujourd'hui RTL) est devenue une radio périphérique privée française en 1933 sous l'égide de la Compagnie luxembourgeoise de radiodiffusion.

4 Sur I'histoire de la télévision en Afrique francophone, voir Tudesq André-Jean (1992), L'Afrique noire et ses télévisions, Paris, Ina/Anthropos; Dioh Tidiane (2009), Histoire de la télévision en Afrique noire francophone, des origines à nos jours, Paris, Karthala.

5 Sur I'histoire de la coopération française en Afrique, voir Goerg Odile et Raison-Jourde Françoise (dir.) (2012), « Les coopérants français en Afrique : portrait de groupe (années 1950-1990) », Cahiers Afrique, 28 ; Goerg Odile et de Suremain Marie-Albane (dir.) (2014), "Coopérants et coopération en Afrique : circulations d'acteurs et recompositions culturelles (des années 1950 à nos jours) », Outre-Mers. Revue d'histoire, 384-385 ; Peraldi Michel (dir.) (2016), « Mobilités et migrations européennes en (post) colonies », Cahiers d'études africaines, 221-222.
} 
a été affecté par l'Ocora. Nous examinerons ensuite son parcours au sein de l'Office de radiodiffusion télévision française (ORTF) (1969-1974), jusqu'à son affectation à FR3 Dom-Tom en janvier 1975 après que celui-ci a été démantelé. À cette seconde période est associé le démarrage de la Télévision sénégalaise à Dakar en 1972, pour lequel Guy Bernède avait été initialement recruté par l'Ocora en 1962. Ce démarrage a lieu alors qu'il est entré dans une nouvelle phase de sa carrière, tant sur le plan institutionnel que matériel puisqu'il devient rédacteur en chef du service d'envoi de programmes de l'ORTF et qu'il se met à utiliser de nouveaux moyens techniques.

Cet article, fondé sur une approche biographique, croise archives et enquête orale et accorde une large place au propre récit de Guy Bernède ${ }^{6}$. Ce dernier a été confronté à d'autres sources, dont les archives que Guy Bernède a lui-même léguées en 2010 aux Archives nationales de France (ANF), témoignant d'un souci de patrimonialisation de son action? ${ }^{7}$ Cette approche a d'une part l'intérêt de nous faire accéder à la trame des acteurs, afin de démêler le " tissu de l'histoire » et d'en comprendre quelques intrigues ${ }^{8}$. Elle nous permet d'autre part, en reconstituant le parcours de Guy Bernède et en identifiant ses ancrages locaux et internationaux, de mieux saisir les circulations de la coopération télévisuelle franco-africaine à cette période et de renseigner un « réseau transnational vécu ${ }^{9}$ ».

\section{L’Ocora Télévision, ou la coopération par hasard (1962-1969)}

\section{De Radio Luxembourg à l'Agence Télévision de l'Ocora}

C'est donc en 1962 que cette histoire commence pour Guy Bernède. Cette année-là en avril, la Société de radiodiffusion de la France d'Outre-mer (Sorafom), qui n'avait pas été restructurée depuis les indépendances, devient le nouvel Ocora ${ }^{10}$. Son directeur général Robert Pontillon, ancien directeur général de la Sorafom, reçoit rapidement une demande d'assistance technique du gouvernement de Fulbert Youlou qui souhaite démarrer une télévision en République du

\footnotetext{
6 Celui-ci a été recueilli dans le cadre d'une série d'échanges informels (conversations téléphoniques et courriers électroniques) en 2019-2020, ainsi que d'un entretien réalisé les 21 et 22 novembre 2019 à son domicile de Boulogne-Billancourt. À cette occasion, certaines des archives qu'il a conservées ont été consultées. Celles-ci sont qualifiées dans l'article d'archives "personnelles » plutôt que «privées » du fait qu'elles contiennent également des documents institutionnels.

7 Voir Bat Jean-Pierre (2012), Le Syndrome Foccart. La politique française en Afrique, de 1959 à nos jours, Paris, Gallimard, p. 48. Le Fonds Guy Bernède aux ANF (28AR), à l'instar de celui de Jacques Foccart, a le statut de " fonds privé » bien qu'il soit en grande partie composé d'archives publiques. Ce legs fut néanmoins louable dans un contexte où les archives des agents du service public audiovisuel, comme celles des hommes politiques, étaient souvent privatisées par leurs détenteurs. Ce fonds, qui contient plusieurs milliers de pages regroupées en 26 cartons (3,8 mètres linéaires), couvre plus de trois décennies de coopération télévisuelle francoafricaine et constitue une mine d'information particulièrement riche sur cette période historique.

${ }^{8}$ Veyne Paul (1971), Comment on écrit l'histoire, Paris, Seuil, p. 51. Je remercie BL pour cette référence.

9 Nicolas Claire (2019), « De l'histoire comparée à la démarche multi-située : sur les traces des cadres sportifs africains », Espaces et Sociétés, 178, p. 55.

${ }^{10}$ La Sorafom avait été créée en janvier 1955 en remplacement de l'ancienne Radiodiffusion de la France d'Outre-mer (RFOM). Cette restructuration menée par Pierre Schaeffer, ingénieur de la Radiodiffusion télévision française et représentant au ministère de la France d'Outre-mer, devait permettre la création de radios « africaines et décolonisées » dans un contexte où les programmes diffusés dans les Territoires africains d'Outre-mer étaient encore très largement produits en France et en français. Sur cette histoire, voir notamment Damome Étienne (2012), «Vers un réseau Outre-mer», in M. Kaltenecker et K. Le Bail (dir.), Pierre Schaeffer. Les constructions impatientes, Paris, CNRS, pp. 164-177.
} 
Congo ${ }^{11}$. En effet, ce dernier s'est laissé convaincre d'acheter une station de marque britannique Pye en début d'année 1962 et veut accélérer le projet ${ }^{12}$.

En octobre 1962, le ministre congolais de l'Information Apollinaire Bazinga se rend à Paris et décision est prise d'organiser trois jours d'émissions expérimentales à l'occasion des fêtes de l'indépendance les 27, 28 et 29 novembre 1962 à Brazzaville. La Sorafom s'était dédiée jusqu'ici à la coopération radiophonique et cet accord donne à la France l'opportunité de créer un service de coopération télévisuelle qui lui permette de s'associer à la création des télévisions nationales dans ses anciennes colonies d'Afrique. Afin de prendre en charge la coopération avec la nouvelle Radiodiffusion télévision congolaise (RTC) et les autres télévisions à venir, Robert Pontillon met sur pied une Agence Télévision, qu'il confie au journaliste Guy Violante. Celle-ci compte deux principaux services : celui des Programmes dirigé par Jacques Canestrier et celui de I'Information dirigé par Jacques Conia ${ }^{13}$.

À cette période, la collaboration de Guy Bernède avec RL s'interrompt brutalement. En 1962, alors que la guerre d'indépendance algérienne prend fin, le contrôle du pouvoir sur I'information est devenu tel que la fronde monte parmi les journalistes. Le jeune Syndicat des journalistes de radio et de télévision proteste contre les atteintes faites à la liberté de la presse et la Radiodiffusion télévision française (RTF) est secouée par plusieurs grèves ${ }^{14}$. À RL la censure s'exerce aussi et Jean Grandmougin, qui faisait l'objet de pressions politiques pour sa liberté de ton, est limogé au nom de prétendus liens avec l'Organisation de l'armée secrète (OAS) ${ }^{15}$. Guy Bernède, après avoir plaidé sa cause, connaît le même sort le 31 octobre 1962.

Titulaire depuis peu d'une carte de réalisateur de la RTF, il se met en quête d'un emploi lorsqu'il est contacté par Guy Violante. Ce dernier, qui cherche encore un journaliste pour assurer les émissions de Brazzaville et a obtenu son contact par le bouche-à-oreille, lui propose un contrat de pigiste. Guy Bernède lui, n'a pas eu d'autre contact avec l'Afrique que quelques reportages qu'il a faits pour RL - en 1958 notamment, il a couvert le lancement d'une fusée depuis le Centre interarmées d'essais d'engins spéciaux à Hammaguir (Algérie) - et deux films sur lesquels il a travaillé à Paris ${ }^{16}$. II n'a pas non plus d'expérience des journaux télévisés (JT) qu'il faut assurer dans le cadre de cette pige mais Guy Violante, à qui il expose le risque, décide de le recruter.

\footnotetext{
11 En 1957, une fois le réseau Sorafom structuré et sa direction devenue un poste politique d'importance, Pierre Schaeffer fut limogé par le ministre de la France d'Outre-mer Gérard Jaquet et remplacé par Robert Pontillon, ancien résistant et ancien directeur adjoint du cabinet de son prédécesseur Gaston Defferre. Voir Schaeffer Pierre (1978), Les Antennes de Jéricho, Paris, Stock, p. 305.

12 Dioh T., Histoire de la télévision..., op. cit., p. 94.

${ }^{13}$ Avant son recrutement par I'Ocora en 1962, Jacques Conia avait vécu en Algérie, où il était resté après son service militaire et s'était formé au journalisme, puis au Cambodge (Fédération indochinoise) où il avait travaillé dans une plantation d'hévéas, une expérience à l'origine de son premier livre Saignée blanche (Denoël, 1955).

${ }^{14}$ Sur ce sujet, voir Bourdon Jérôme (2014 [1990]), Histoire de la télévision sous de Gaulle, Paris, Presses des Mines, pp. 107-142.

15 Sur ce sujet, voir Lauzanne Bernard (1992), «L'affaire Grandmougin : comment fut contraint au silence "I'homme le plus écouté de France" ", Cahiers d'histoire de la radiodiffusion, 35, pp. 54-60bis; Grandmougin Jean (1967), Lettre ouverte au Ministre de I'Information, Paris, Albin Michel.

16 // est minuit, docteur Schweitzer d'André Haguet (1951) et Mon frangin du Sénégal de Guy Lacourt (1953).
} 


\section{Les émissions expérimentales de Brazzaville : un tremplin vers la coopération}

Guy Bernède s'envole donc pour Brazzaville en novembre 1962, accompagné notamment du réalisateur Claude Augot, où ils rejoignent la première partie de l'équipe. Comme il l'explique, le milieu de la coopération lui est alors inconnu:

Moi la coopération je ne savais pas ce que c'était avant, quand j'étais à [Radio] Luxembourg. C'était vraiment autre chose, un autre milieu. Et puis la coopération j'ai vu ce que c'était, ce dont certains pauvres pays avaient besoin. Je me souviens [de] Brazzaville avec les quartiers populaires, Poto-Poto, Bacongo... ${ }^{17}$

Sur place, le dispositif n'est pas encore opérationnel et l'Agence Télévision a commandé du matériel français pour compléter la station Pye, prévue pour la télévision scolaire, qui arrive après l'équipe par avion militaire ${ }^{18}$. Louis Ménard, ingénieur du Service technique de l'Ocora, est chargé de mettre sur pied la station dans un ancien bâtiment du Service des Instruments de Mesure, avec le concours d'une équipe congolaise recrutée parmi les techniciens de Radio Congo. Guy Bernède lui, enregistre les allocutions de Fulbert Youlou et Apollinaire Bazinga qui ouvriront les émissions, et réalise quatre reportages avec son équipe ${ }^{19}$. Une seconde emmenée par Claude Augot filme aussi et, en plus de ces tournages, Jacques Canestrier envoie des éléments de Paris $^{20}$. Dans le même temps, 16 postes de télévision sont installés dans Brazzaville pour des retransmissions publiques, notamment au Centre culturel français et dans les locaux de la Compagnie générale de télégraphie sans fil (CSF), et des mires de la RTC sont créées ${ }^{21}$.

\footnotetext{
17 Entretien avec Guy Bernède, 21 et 22 novembre 2019, Boulogne-Billancourt. Sur les représentations des coopérants, voir, pour la période plus récente: Quashie Hélène (2016), «Débuter sa carrière professionnelle en Afrique. L'idéal d'insertion sociale des volontaires français à Dakar et Antananarivo (Sénégal, Madagascar) ", Cahiers d'études africaines, 221-222, pp. 53-80.

18 Celui-ci comprend notamment des caméras et télécinémas de marque Thomson et un laboratoire de développement de marque Debrie.

${ }^{19}$ Ces reportages, qui portent sur l'école des peintres de Poto-Poto, I'Institut de recherche scientifique du Congo, l'Institut Pasteur de Brazzaville et le fleuve Congo, sont développés et montés sur place.

${ }^{20}$ Ceux-ci comprennent un reportage réalisé par Guy Bernède avant le départ sur I'Institut national des sports de Paris, où s'entrainent des stagiaires congolais, des archives de la RTF datant des fêtes de l'indépendance de 1960 que Guy Bernède a réussi à récupérer et six programmes récréatifs : trois dessins animés (Pour le meilleur et pour le pire, Analyatou-Bari et Simbad le marin) et trois films (Charlot somnambule, Le Dragon de Guy Lefranc et Si tous les gars du monde de Christian-Jaque, ce dernier finalement remplacé par son autre film François ler, prêté par le Centre culturel français de Brazzaville).

${ }^{21}$ Images fixes diffusées avant les programmes pour permettre le réglage des téléviseurs, les mires concourraient à l'identité visuelle des chaînes qui les utilisaient aussi après l'arrêt des programmes, durant des incidents techniques ou en cas de grève. Les mires de la RTC (et celles des autres télévisions africaines qui solliciteront ensuite l'assistance de l'Ocora) avaient été réalisées sur le modèle de la mire à quadrillages française dite « au cheval de Marly », adoptée par la RTF en 1953 puis reprise par l'ORTF en 1964.
} 
Illustration $\mathrm{n}^{\circ} 1:$ Mire de la RTC 22

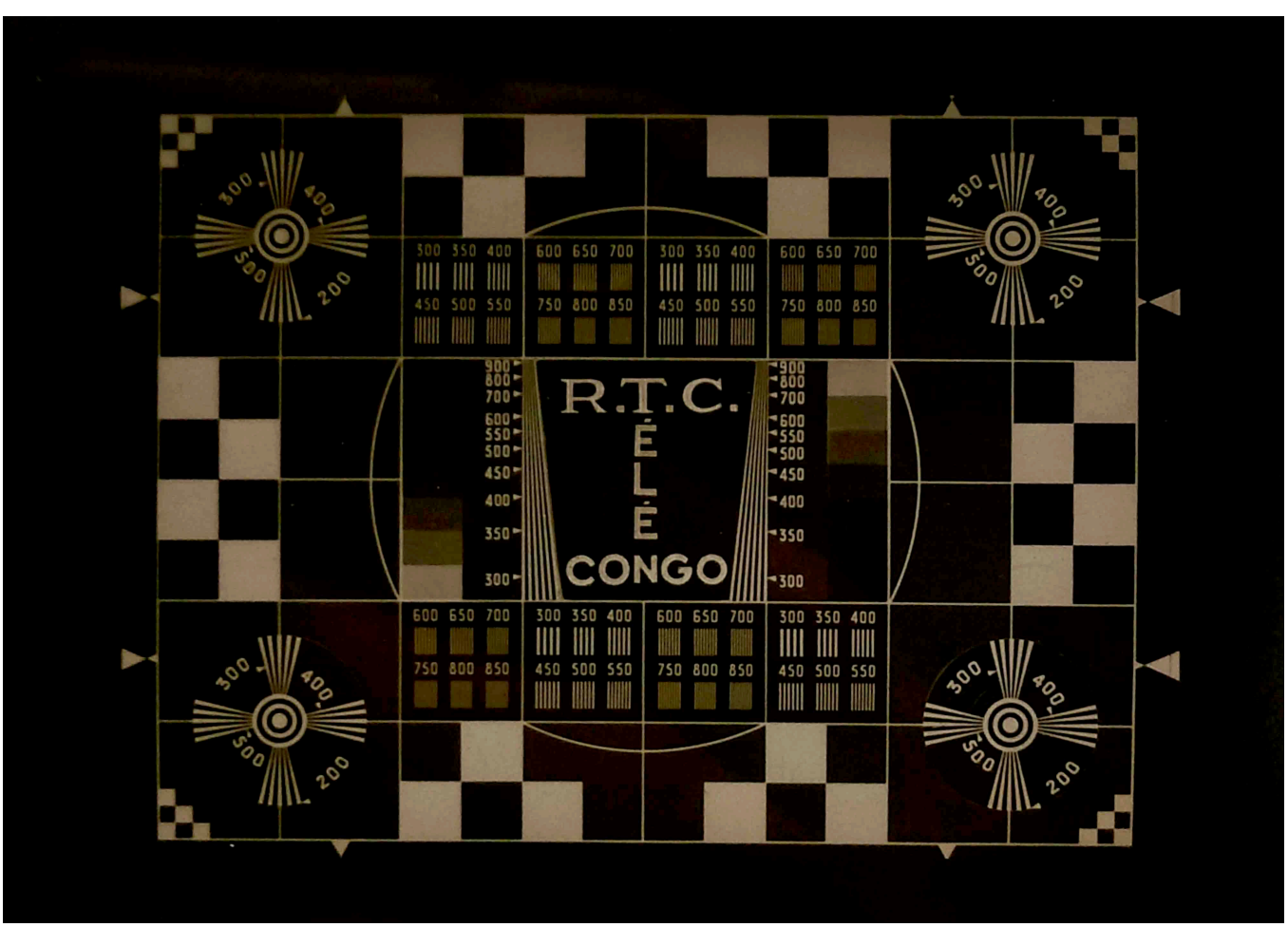

Tout est prêt et le programme, qui précise que les émissions sont le fruit d'un " effort solidaire associant les techniciens français et congolais", est diffusé aux officiels ${ }^{23}$. Le 27 novembre 1962, les émissions démarrent comme prévu avec les fêtes nationales. Programmées de $20 \mathrm{~h} 30$ à $23 \mathrm{~h} 30$ les trois jours, elles représentent neuf heures de service à assurer. Les films de l'Ocora vont être diffusés, mais la RTC va aussi produire de premières émissions en direct vidéo depuis le plateau : du « folklore congolais » d'une part, qui occupe une place importante dans la programmation (orchestre Negro Jazz, chorale des Piroguiers, ballets Diaboua); les premiers JT nationaux d'autre part, soit une édition de vingt minutes les trois jours et une édition de fin de cinq minutes les deux premiers jours. L'équipe du JT est française, à l'exception de Bernard Bakabadio, projectionniste du ministère de I'Information, mais l'Ocora et la RTC conviennent qu'un binôme congolais-français assurera sa présentation, afin d'afficher une égalité sur le plateau. Celui-ci est présenté par le journaliste-vedette François Itoua, rédacteur en chef de Radio Congo et directeur adjoint de la nouvelle RTC, en binôme avec Jacques Conia ou Guy Bernède en alternance : «On était trois journalistes, Conia et moi pour le côté français, et Itoua. II y en avait deux qui étaient en plateau, un Blanc un Noir. Et I'autre Blanc [faisait] les commentaires des films d'actualité24 ».

\footnotetext{
22 ANF, 28AR/2, dossier Congo.

23 Archives personnelles de Guy Bernède, Programme des émissions de télévision des 27-28-29 novembre 1962.

24 Entretien avec Guy Bernède, 21 et 22 novembre 2019, Boulogne-Billancourt. Ces films sont ceux tournés par l'Ocora à Brazzaville et envoyés de Paris, et ceux fournis par l'agence britannique Visnews (voir ci-après).
} 
Illustration $n^{\circ} 2$ : Guy Bernède et François Itoua sur le plateau de la RTC, Brazzaville, novembre $1962^{25}$

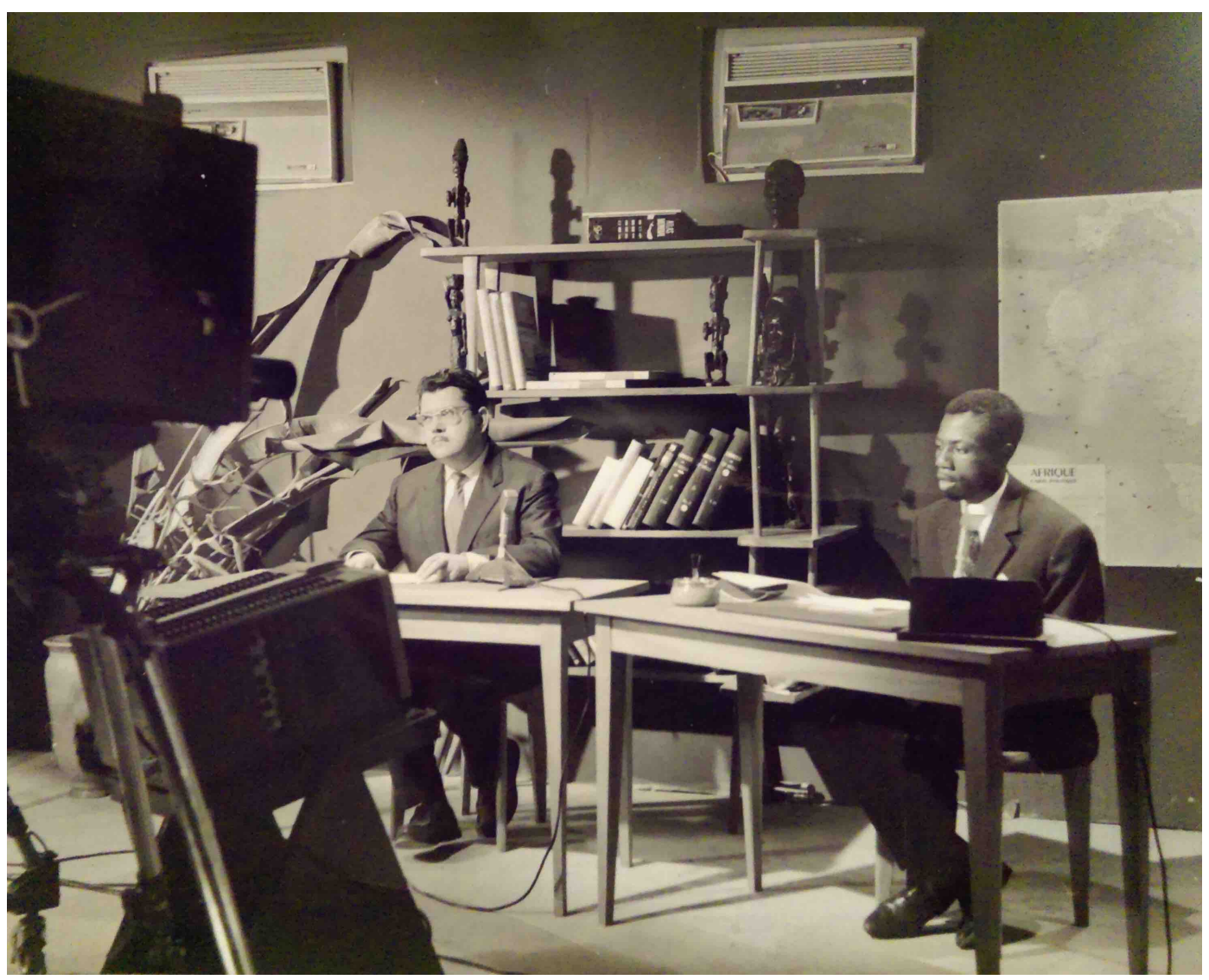

Les journées expérimentales, bien qu'accessibles à un public très restreint (essentiellement les membres de la haute administration, de l'élite économique et les expatriés) sont un succès technique ${ }^{26}$. Le succès est aussi politique et le 30 novembre 1962, l'équipe de l'Ocora est décorée par le ministre Bazinga, avant le départ pour Paris.

Guy Bernède, dont la pige prend fin avec l'arrêt des émissions, ne veut pas rentrer en France. Par ailleurs, son ami et ancien collègue de RL Pierre Aymard est maintenant assistant technique à Radio Côte d'Ivoire et, après l'avoir contacté, il part pour Abidjan où il doit faire escale deux jours. Sur place, il décide de prolonger sa route jusqu'à Dakar, qu'il aimerait découvrir, et Pierre Aymard, qui a été en poste à Radio Dakar avant l'indépendance, lui communique un contact à Radio Sénégal (ex-Radio-Inter-AOF). Alors qu'il se rend visiter leurs locaux, Guy Bernède est informé que Guy Violante, qui a appris qu'il est à Dakar, a laissé un message pour lui : il arrive le lendemain et veut absolument le voir.

\footnotetext{
25 Archive personnelle de Guy Bernède.

${ }^{26}$ En effet, le prix d'un téléviseur s'élève alors à environ 130000 Francs CFA (FCFA) au Congo, un investissement impossible à réaliser pour la majorité des travailleurs. Voir Bekombo Manga (1966), «Brazzaville à l'heure de la télévision congolaise », Revue française de sociologie, 7(2), p. 192. Cette inégalité d'accès à l'équipement, qui concerne au départ tous les États, incite ces derniers à développer les retransmissions publiques avec la construction de «cases d'écoute » en Haute-Volta et au Gabon, de " téléclubs » au Sénégal, ou de "halls d'information" en Côte d'Ivoire par exemple. Voir Dindané Ousmane (2016), De l'arrière du décor. Une odyssée au cceur de la Télévision ivoirienne, Abidjan, L’Encre Bleue, pp. 22-24.
} 
Le démarrage manqué de la Télévision sénégalaise, opportunité d'un avancement de carrière

Dès l'indépendance du Sénégal en 1960, le président Léopold Sédar Senghor avait en effet souhaité créer une télévision, dont le démarrage est prévu le 24 décembre $1962^{27}$. Une équipe sénégalaise a été constituée et un studio aménagé à Radio Sénégal, que Guy Bernède visite. Le Sénégal a aussi sollicité l'Ocora et Guy Violante, convaincu par la prestation de Guy Bernède à Brazzaville, lui propose de piloter la future équipe d'assistance technique. Au moment où les deux hommes s'entretiennent, le pays est toutefois en proie à une grave crise politique opposant le président de la République Senghor et le président du Conseil Mamadou Dia, et les dates de la mission ne sont pas déterminées ${ }^{28}$. Guy Bernède, qui par ailleurs ne se projette pas dans la coopération, décline l'offre. Devant l'insistance de Guy Violante, il passe néanmoins voir Jacques Conia à son retour à Paris, qui le convainc d'accepter. N'ayant aucune certitude d'obtenir un poste de réalisateur à la RTF comme il le voudrait, Guy Bernède signe alors le contrat. Mais la crise politique au Sénégal ne se résout pas, bien au contraire, et Mamadou Dia est arrêté et incarcéré. Le démarrage de la télévision, déjà loin de susciter l'unanimité du gouvernement sénégalais, notamment du fait de son coût pour le pays, n'est plus une priorité et l'accord avec l'Ocora est suspendu' ${ }^{29}$.

Guy Bernède, qui dispose d'un contrat, décide de faire valoir ses droits. Guy Violante propose alors de l'affecter à Brazzaville où les autorités souhaitent maintenant mettre en place un service permanent de télédiffusion. Guy Bernède, qui explique ne pas avoir envisagé une expatriation, refuse encore, si bien que Guy Violante lui propose de devenir son adjoint « en charge des études et des démarrages ». II précise qu'il devra toutefois retourner à Brazzaville dans ce cadre, afin de mettre en place les premières émissions prévues pour mars 1963. Avec ces conditions, Guy Bernède accepte et repart en février 1963 pour le Congo où il assure la représentation de l'Ocora Télévision auprès du directeur de la RTC Jean Malonga et de François Itoua, devenu directeur de Télé Congo ${ }^{30}$.

Il doit également s'occuper de recruter une équipe congolaise dans un contexte où I'Ocora, qui a hérité du Studio-école de la Sorafom, forme maintenant les professionnels de télévision africains ${ }^{31}$. À la demande des gouvernements congolais et gabonais, son directeur André Clavé s'est d'ailleurs rendu en mission à Brazzaville et Libreville en 1962 afin de recruter des stagiaires ${ }^{32}$. La formation dure toutefois plusieurs mois, en France, et ceux-ci ne pourront

\footnotetext{
27 Dioh T., Histoire de la télévision..., op. cit., p. 187.

28 Sur ce chapitre de I'histoire politique du Sénégal, voir notamment Colin Roland, Perrot Thomas et Smith Étienne (2010), " "Alors, tu ne m'embrasses plus Léopold ?" Mamadou Dia et Léopold S. Senghor ", Afrique contemporaine, 233, pp. 111-132. Voir aussi l'article de Mouhamadou Moustapha Sow sur le traitement médiatique de la crise dans ce numéro.

29 D'après A.-J. Tudesq, le gouvernement sénégalais avait, avant même le déclenchement de la crise, exprimé ses réticences. II rapporte en effet que lors du Conseil des ministres du 28 novembre 1962, seuls trois de ses membres se seraient montrés favorables au démarrage. Voir Tudesq A.-J., L'Afrique noire..., op. cit., p. 51.

30 Pour une correspondance entre Guy Bernède, Guy Violante, Jacques Conia et Jacques Canestrier à cette période, voir ANF, 28AR(2), dossier Congo, mars-juin 1963.

31 Le Studio-école de la Radiodiffusion d'Outre-mer avait été créé en 1955 par Pierre Schaeffer afin de former les professionnels de radio africains (techniciens et journalistes). Installé au Pavillon de la Muette en forêt de Saint-Germain-en-Laye (Maisons-Laffitte), il constituait I'un des piliers de la nouvelle Sorafom.

32 Ancien résistant et proche de Pierre Schaeffer qui l'avait nommé, André Clavé est resté directeur du Studio-école après le limogeage de Pierre Schaeffer en 1957 et jusqu'à sa dissolution en 1969 (voir ci-après).
} 
assurer le démarrage des émissions. Guy Bernède, qui a accès aux dossiers des candidats, décide de constituer une équipe parmi les recalés :

On a pris sur place ceux qui n'avaient pas été acceptés, [...] pour être caméramans vidéo, assistants monteurs, etc. [...] Ce qui a posé un problème, parce que quand les premiers stagiaires formés à Paris sont arrivés, ils étaient moins bons que ceux qui avaient été formés sur place. [...] Ceux qui faisaient bien le boulot, c'était ceux qu'on avait formés. II fallait le reconnaître. Après d'ailleurs Clavé [l']a reconnu. ${ }^{33}$

L'Ocora fournit déjà des programmes à Radio Congo et une convention de fourniture de programmes télévisés est rapidement signée pour Télé Congo. Celle-ci prévoit l'envoi par fret aérien de cinq heures hebdomadaires, comprenant trente minutes d'informations extraites des JT de la RTF et de tournages dédiés. Dès le mois d'avril 1963, la RTC passe d'un à trois jours d'émissions par semaine, tandis qu'un autre démarrage s'organise dans le Gabon voisin. Léon Mba, qui a lui aussi acquis une station Pye, a contacté l'Ocora ${ }^{34}$. Comme Guy Bernède est déjà dans la région, Guy Violante le détache à Libreville où il retrouve Louis Ménard, chargé d'aménager le futur studio dans les locaux de Radio Gabon. Le démarrage a lieu le 9 mai 1963 et une convention identique à celle du Congo est signée.

Télé Congo et Télé Gabon étant lancées, Guy Bernède circule durant deux mois entre les deux capitales afin d'assurer leur suivi. Lorsqu'il rentre à Paris, deux autres télévisions nationales sont sur le point de démarrer, en Haute-Volta et en Côte d'Ivoire, mais il n'est pas impliqué sur ces projets ${ }^{35}$. II s'occupe en revanche d'un autre dossier: le déménagement de l'Agence Télévision.

\section{La création du centre de production de Courbevoie}

En début d'année 1963, Guy Violante a trouvé un bâtiment avec un grand hangar à Courbevoie afin de créer un véritable centre de production. À l'automne, l'équipe emménage alors que les moyens techniques ne sont pas complètement installés car le besoin est devenu pressant ${ }^{36}$.

Après la réussite des journées de Brazzaville, que l'équipe surnomme désormais les «Trois Glorieuses » en clin d'œil aux journées insurrectionnelles des 13, 14 et 15 août 1963, un nouveau Service technique a en effet été créé afin de prendre en charge les aspects matériels des futurs démarrages ${ }^{37}$. En outre, l'Agence fournit maintenant quatre télévisions en programmes (Télé Congo, Télé Gabon, Voltavision et la Télévision ivoirienne), soit une base de quatre-vingts heures mensuelles. Leur circulation entre la France et l'Afrique implique une certaine logistique, d'autant que les vols ne sont pas nombreux et que seules deux compagnies

\footnotetext{
33 Entretien avec Guy Bernède, 21 et 22 novembre 2019, Boulogne-Billancourt. Guy Bernède connaissait André Clavé, qu'il avait rencontré en 1951 sur le tournage du film « II est minuit Docteur Schweitzer », adapté de sa pièce originale. II explique toutefois que l'Agence Télévision (alors située avenue Hoche à Paris) et le Studio-école, géographiquement distants, étaient cloisonnés.

34 Dioh T., Histoire de la télévision..., op. cit., p. 119.

35 Ibid., pp. 47 et 105 .

36 Les travaux réalisés ont déjà permis d'aménager le hangar, qui contient un studio équipé de caméras mixtes vidéo-cinéma Thomson, un laboratoire de développement, une salle de tirage, des salles de montage et des bureaux. Un auditorium a aussi été créé pour le son, ainsi qu'une cinémathèque pour le stockage des films.

37 Sur les circonstances et impacts des «Trois Glorieuses », voir Kiriakou Héloïse (2019), "Ébranler la gérontocratie au pouvoir : quand les cadets font la révolution au Congo-Brazzaville », in Y. Denéchère (dir.), Enjeux postcoloniaux de l'enfance et de la jeunesse. Espace francophone (1945-1980), Bruxelles, Peter Lang, pp. 192-202.
} 
opèrent sur ces lignes (Air Afrique et I'Union de Transports Aériens). Ces programmes, à l'exception des trente minutes d'actualités hebdomadaires, sont alors regroupés par le Service d'envoi qui les achemine une à deux fois par mois jusqu'à l'aéroport du Bourget afin qu'ils soient expédiés aux stations. II ne s'agit toutefois que de prêts, les films devant être réexpédiés à Courbevoie après leur diffusion. Bien que Guy Bernède dise ne plus avoir de souvenirs à ce sujet, ses archives font état de nombreux problèmes dans ces retours. ${ }^{38}$

En décembre 1963, alors que le centre se rôde, la RTF inaugure sa nouvelle Maison de la

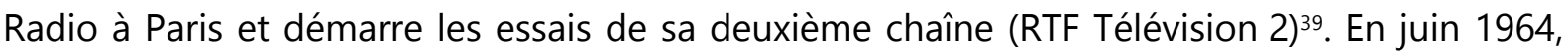
dans le cadre de la réforme du service public de radio-télévision, elle est remplacée par I'ORTF40. Pour l'Ocora les impacts sont minimes et l'Agence Télévision reprend ses images comme elle le faisait avec la RTF avant lui. Toutefois, Robert Pontillon est remplacé par François Cardin, ancien résistant lui aussi, sous le mandat duquel plusieurs choses changent. Les installations de Courbevoie sont notamment commercialisées (une tâche confiée à Guy Bernède) et le Studio-école est réformé en 1965 afin de ne former que des cadres moyens et supérieurs, les «simples agents d'exécution » étant désormais formés à l'échelon local ${ }^{41}$. Comme l'explique Guy Bernède :

Au départ [on envoyait] une équipe de dix-douze, avec un réalisateur, un JRI [journaliste reporter d'images], un opérateur de prise de vue, un monteur, un technicien de laboratoire, un technicien vidéo, un preneur de son... Puis après le preneur de son on l'a rapidement supprimé parce qu'il y avait des preneurs de son à la Radio [nationale], donc il suffisait de les former pour la télévision. Ça se faisait très très vite, sans passer par le Studio-école !42

Depuis 1963, aucun démarrage ne s'est concrétisé et l'Agence se dédie à l'envoi des programmes et à l'assistance aux quatre stations ${ }^{43}$. Guy Bernède continue néanmoins de séjourner en Afrique, à l'occasion de réunions de I'Union des radiodiffusions et télévisions nationales d'Afrique (Urtna) ou pour les Jeux africains de Brazzaville en 1965 par exemple.

En 1966, il s'occupe du démarrage de la Télévision congolaise à Kinshasa, le plus difficile selon lui. La République démocratique du Congo (RDC), ancienne colonie belge, a en effet acheté son matériel à la Radio Corporation of America, impliquant la présence de coopérants belges et états-uniens. Guy Bernède fait état de tensions dans ce cadre (choix techniques, programmes) et dira : "C'est la seule [télévision] où au départ, il y avait quand même des étrangers [personnels ni congolais ni français, ndla]. Ça a été le plus difficile, de faire vivre tous

\footnotetext{
38 On retrouve en effet dans sa correspondance de nombreuses notes portant sur des sacs éventrés, des bordereaux illisibles, des problèmes de douanes. Un problème récurrent est aussi celui des frais de réexpédition trop élevés, dont les télévisions africaines ont du mal à s'acquitter. Voir ANF, 28AR(2/3).

${ }^{39}$ Rappelons en effet que la télévision et ses métiers sont, de la même façon, en pleine structuration en France.

${ }^{40}$ Sur ce processus, voir Bourdon J., Histoire de la télévision..., op. cit., pp. 53-75.

41 Anonyme, "La coopération radiophonique entre la France et l'Afrique », Le Monde, 5 février 1965. En ligne. URL: https://www.lemonde.fr/archives/article/1965/02/05/la-cooperation-radiophonique-entre-la-france-et-l-

afrique_2183897_1819218.html (consulté le 29 septembre 2020).

42 Entretien avec Guy Bernède, 21 et 22 novembre 2019, Boulogne-Billancourt. II précise que ces techniciens étaient le plus souvent formés au maniement d'un appareil spécifique.

43 En 1965 par exemple, elle conçoit le nouveau bâtiment de la RTC. Voir ANF, 28AR(2), Plan du bâtiment de la télévision, RTC Brazzaville, avril 1965.
} 
ces gens ensemble ${ }^{44} »$. En 1967, il est chargé du démarrage de la Télévision malgache avec Louis Ménard, juste avant les événements de Mai $68^{45}$. Malgré les négociations, menées par Guy Bernède, pour défendre I'Ocora, la restructuration ne peut être évitée.

\section{Dans les méandres de la coopération : la Daec (1969-1974)}

\section{De l'Agence Télévision de l'Ocora à la Télévision extérieure de l'ORTF}

En effet, en juillet 1969, l'Ocora est fusionné avec la Direction des relations extérieures de I'ORTF, qui s'occupait des rapports avec les autres radiotélévisions étrangères (non africaines). Ensemble, ils forment la nouvelle Direction des affaires extérieures et de la coopération (Daec), à la tête de laquelle est nommé Raymond Poussard, ancien directeur adjoint des Affaires culturelles et techniques du Quai d'Orsay et nouveau directeur adjoint de I'ORTF ${ }^{46}$. Grosse machine, la Daec emploie environ cinq-cents personnes et compte plusieurs départements, dont les Affaires internationales, la Coopération, la Radio extérieure et la Télévision extérieure ${ }^{47}$. Son objectif, d'après Raymond Poussard :

Assurer la présentation de la France sous tous ses aspects sur les ondes du monde entier, soit qu'elle émette elle-même, soit qu'elle fournisse des programmes aux autres radiodiffusions. [...] [Et] assurer la coopération technique, non seulement avec les États africains et malgache, mais avec tous les pays du monde. ${ }^{48}$

Cette fois, la restructuration a des implications concrètes pour le personnel de l'Ocora. Le Studio-école est fermé, la formation étant dorénavant assurée par l'ORTF. Une large partie du personnel est toutefois repris, dont les ingénieurs qui, à l'instar de Louis Ménard, sont affectés à la Direction technique. Les journalistes et techniciens aussi sont pour la plupart affectés à la Radio extérieure ou à la Télévision extérieure ${ }^{49}$. C'est le cas de Guy Bernède, contraint de quitter Courbevoie pour la Maison de la Radio:

[C'était] un beau petit centre où on pouvait tout faire. On tournait le film, on le développait, on le montait, on le sonorisait, on faisait des copies. [...] C'était un petit bijou pour eux, alors ils ont sauté dessus. Mais les gens qui étaient là, on les a vidés, je parle des responsables. [...] Les monteurs tout ça, eux ils sont restés. Ils n'étaient pas très nombreux [et] comme l'ORTF reprenait I'outil, il reprenait en même temps ceux qui faisaient marcher l'outil. ${ }^{50}$

\footnotetext{
44 Entretien avec Guy Bernède, 21 et 22 novembre 2019, Boulogne-Billancourt. Cette citation, loin d'être anecdotique, montre à quel point les nouvelles télévisions d'Afrique francophone étaient alors considérées comme relevant du pré-carré français, étendu ici aux anciennes colonies belges.

${ }^{45}$ Sur la crise et ses impacts à l'ORTF, voir Bourdon J., Histoire de la télévision..., op. cit., pp. 285-314.

46 Anonyme, «M. Raymond Poussard directeur général adjoint de I'ORTF », Le Monde, 30 janvier 1969. En ligne. URL: https://www.lemonde.fr/archives/article/1969/01/30/m-raymond-poussard-directeur-general-adjoint-de-l-o-r-tf_2423806_1819218.html (consulté le 17 septembre 2020).

$\overline{47}$ La Daec comprend également un Service enseignement, dont la division Enseignement à l'étranger est dirigée par Yves le Gall, ancien de l'Ocora Radio et artisan de la Télévision scolaire du Niger démarrée en 1964, ainsi que du Programme d'éducation télévisuelle adopté en 1969 par l'Assemblée nationale de Côte d'Ivoire.

48 ORTF, "Des ondes courtes à la mondovision: la DAEC», Micros et caméras, 25 octobre 1969. En ligne. URL: https://www.ina.fr/video/CPF86625975 [00:02:26-00:03:07] (consulté le 23 mai 2017).

${ }^{49}$ Les professionnels de ces services se connaissent souvent depuis longtemps et sont parfois amis. Guy Bernède raconte par exemple que le directeur de la Télévision extérieure Lucien Renault et son administrateur Albert Bazire avaient été en poste ensemble à RadioBrazzaville après la Seconde Guerre mondiale.

50 Entretien avec Guy Bernède, 21 et 22 novembre 2019, Boulogne-Billancourt.
} 
Pour lui, les télévisions d'Afrique sont perdantes car le centre qui leur était dévolu doit maintenant fournir toutes les télévisions étrangères, notamment les européennes ayant un fort besoin de programmes. En outre, les tâches qu'il se retrouve devoir effectuer sont éloignées de son métier ${ }^{51}$. Rapidement, la Daec est toutefois saisie d'un ancien dossier de l'Ocora : le Sénégal, toujours présidé par Senghor, veut relancer son projet de télévision.

\section{Le redémarrage de la Télévision éducative à Dakar}

En 1964, après la suspension du projet de télévision nationale, une Télévision éducative avait été créée à Dakar dans le cadre d'un projet pilote de l'Unesco. Celle-ci était exploitée dans les installations de Radio Sénégal prévues pour la télévision nationale, celles qu'avait visitées Guy Bernède en 1962. En janvier 1970, ces émissions, trop coûteuses, sont interrompues et Radio Sénégal souhaite reprendre la station ${ }^{52}$. Comme le commente Guy Bernède: "Ils [le gouvernement sénégalais] voulaient leur télévision, d'autant plus qu'il y avait toutes [...] [les autres. Et] Senghor, qui avait voulu être le premier, n'était maintenant pas le dernier mais presque, alors c'était un peu gênant ${ }^{53}{ }^{\prime \prime}$.

Comme le Sénégal dispose déjà d'un début d'infrastructure, la demande d'assistance échoit directement au Service ingénierie ${ }^{54}$. Sandor Polgar, un ancien de I'Ocora que Guy Bernède connaît bien, est dépêché à Dakar en septembre 1970 afin d'évaluer les installations et les investissements nécessaires. Au total, les seuls besoins en équipement, à acheminer de France, sont alors estimés à 43 millions de FCFA hors droits de douane ${ }^{55}$.

Ces recommandations sont transmises au gouvernement sénégalais, qui constitue en janvier 1971 un Groupe de travail sur l'avenir de la télévision éducative ${ }^{56}$. Aussitôt, Honorat Ayande, ancien chef du Service technique de la Télévision éducative, est mandaté pour réévaluer l'infrastructure ${ }^{57}$. De janvier à avril, 33 réunions sont organisées et 38 personnalités consultées $^{58}$. À l'issue de cette phase, le Groupe de travail remet un rapport de synthèse dans lequel il recommande la fusion de Radio Sénégal et de la Télévision éducative ${ }^{59}$. II relève néanmoins le fait que celle-ci ne peut intervenir qu'à certaines conditions techniques,

\footnotetext{
51 S'il continue dans un premier temps de travailler sur le démarrage de la Télévision camerounaise, auquel il avait été affecté avec Louis Ménard juste avant la fusion, la première mission qui lui est confiée consiste en effet à produire une étude sur le Service commercial géré par la Télévision extérieure.

$52 \mathrm{ANF}, 28 \mathrm{AR}(3)$, note préliminaire pour un nouveau démarrage de la télévision éducative à Dakar, septembre 1970, p. 1.

53 Entretien avec Guy Bernède, 21 et 22 novembre 2019, Boulogne-Billancourt.

54 Ce service était I'un des trois services du Service technique de la Daec, qui avait pour charge d'effectuer tous les travaux d'études techniques et de fournitures demandés par les organismes étrangers pour la création et le développement de leurs réseaux.

55 ANF, 28AR(3), note préliminaire..., rap. cité. Une liste est jointe en annexe, sur laquelle on peut voir que de l'ancien matériel de la RTF est recyclé, et que des récepteurs «tropicalisés » sont fournis pour la réception.

56 Rattaché au ministère de l'Information, il est composé de cinq membres : le conseiller du ministre Claude Choin, le directeur général de Radio Sénégal Alioune Fall, le directeur de la Radio éducative Mamour Dioum, le coordinateur des moyens audiovisuels à l'Office national de coopération et d'assistance au développement Boubacar Sock, et l'expert en radios et télévisions éducatives de I'Unesco Michel Bourgeois.

57 ANF, 28AR(3), note à l'attention du Groupe de travail chargé d'étudier les problèmes de la Télévision, 14 janvier 1971.

58 Parmi elles l'ancien chef de la Télévision éducative Ibrahima Ba, le secrétaire général de Radio Sénégal Lamine Hane, le chef du Service cinéma de la Direction de I'Information du ministère Paulin Vieyra, des agents de I'ORTF (Sandor Polgar, Yves le Gall), des représentants de I'Unesco, de I'OMS, du Pnud, de I'Unicef et du BIT, ou encore les conseillers culturels des ambassades de quatre " pays amis » (France, Belgique, Japon et Canada).

${ }^{59} \mathrm{ANF}, 28 \mathrm{AR}(3)$, rapport du Groupe de travail sur l'avenir de la télévision éducative, 27 avril 1971.
} 
financières et de personnel et que « des missions d'experts pourraient être sollicitées dans le cadre de l'assistance technique bilatérale et internationale ${ }^{60} »$.

Le Groupe de travail est par ailleurs d'avis d'associer télévisions éducative et généraliste, une position qui n'est pas partagée par tous ${ }^{61}$. En effet, trois ambassades (Belgique, Japon, Canada) et les cinq organisations intergouvernementales consultées (Unesco, OMS, Pnud, Unicef, BIT) pensent que la télévision doit rester éducative. L'ambassade de France, elle, préfère l'autre option et se dit même "disposée à appuyer une demande d'aide ${ }^{62}$ ». En avril 1971, Sandor Polgar retourne à Dakar et rencontre le ministre de I'Information Ousmane Camara, qui lui demande qu'un spécialiste des programmes soit envoyé. À lui aussi, I'ambassade de France dit qu'elle « est très favorable à ce projet qui dotera le Sénégal d'une télévision culturelle, avec des programmes ORTF et des émissions réalisées sur place, suivant les possibilités locales ${ }^{63}$ ". En mai 1971, Ousmane Camara et le directeur de Radio Sénégal Alioune Fall se rendent à Paris pour trouver un accord sur les termes de cette coopération, après quoi le ministre annonce préparer un projet de loi pour la création d'une Radiodiffusion télévision nationale ${ }^{64}$.

Guy Bernède est saisi du dossier et se rend à Dakar en juin 1971, où il rencontre le ministre, des membres du Groupe de travail, le rédacteur en chef de Radio Sénégal Racine Sy, le conseiller technique du président Senghor M. Richard, ainsi que les équipes de l'ambassade de France et de la Mission d'aide et de coopération (Mac) ${ }^{65}$. De retour à Paris, il définit les conditions du démarrage, qui incluent l'utilisation de programmes importés (en français) et le détachement de sept assistants techniques ${ }^{66}$. Soulignant que les émissions attractives ne sont pas nécessairement distractives, il rejoint par ailleurs le point de vue des autorités françaises :

Certains ne voudraient voir qu'une télévision éducative : ils ne se rendent pas compte que le Sénégal n'a pas les moyens de mettre en place et de développer une télévision de ce type - les émissions éducatives ne peuvent être réalisées que localement et nécessitent des moyens importants [alors que le reste peut être importé, de I'ORTF par exemple]. ${ }^{67}$

Depuis Paris, Guy Bernède reste en contact avec Radio Sénégal. En atteste sa correspondance de juillet-août 1971, dans laquelle il cherche à régler un problème lié à une

\footnotetext{
60 Ibid., p. 10.

61 La question du choix entre télévision éducative et télévision généraliste (parfois qualifiée de « récréative » ou « distractive ») se pose à cette période dans d'autres pays comme le Niger, souvent en lien avec le problème du ratio produits culturels étrangers/production nationale, tous deux liés à la capacité de production locale. Voir ANF, 28AR(3), document sur la télévision récréative [Niger], décembre 1973.

${ }^{62} \mathrm{ANF}, 28 \mathrm{AR}(3)$, rapport du Groupe..., rap. cité, p. 6. Elle annonce par ailleurs que la France est disposée à octroyer 150 millions de FCFA via le Fonds d'aide et de coopération ( $\mathrm{Fac}$ - sur les 162,5 millions estimés pour la couverture totale du territoire en 1975 - et va bientôt fournir, via I'ORTF, une quinzaine de récepteurs transistorisés à piles pour la réception collective dans les villages non électrifiés.

${ }^{63}$ ANF, 28AR(3), compte-rendu d'une mission effectuée du 6 au 12 avril au Sénégal par Monsieur Sandor Polgar du Service de I'Ingénierie, avril 1971, p. 2.

${ }^{64}$ ANF, 28AR(3), exposé liminaire. Rapport du ministre de I'Information Ousmane Camara, Conseil interministériel sur la télévision, Ministère de l'Information chargé des relations avec les Assemblées, n.d. L'Office de radiodiffusion télévision du Sénégal (ORTS) n'est finalement créé qu'en décembre 1973.

${ }^{65}$ Titulaire d'un ordre de mission du ministère de la Coopération, Guy Bernède est en effet tenu de s'entretenir avec l'équipe de la Mac à Dakar.

${ }^{66} \mathrm{ANF}, 28 \mathrm{AR}(3)$, compte-rendu de la mission effectuée à Dakar du 14 au 30 juin 1971 par M. Guy Bernède à titre d'expert télévision, 1er juillet 1971, p. 5-6.

67 Ibid., p. 2. Les crochets sont dans le texte original.
} 
développeuse de films de marque Debrie ${ }^{68}$. Plus généralement, ses archives font état d'échanges réguliers avec les constructeurs et interrogé sur ces relations, il précise :

Il y avait un marché [africain], alors les sociétés avaient intérêt à faire un matériel correct [«tropicalisé »] pour emporter le marché69. [...] On privilégiait le matériel français à un matériel étranger, ça c'est certain, puis quand il n'y avait pas le matériel français qui convenait on prenait autre chose. C'est comme en radio avec le fameux Nagra, il n'y avait pas l'équivalent français [alors] on prenait du matériel suisse ${ }^{70}$.

Dans le même temps, une collaboration s'engage avec Sandor Polgar, avec qui il doit produire une étude préliminaire. Dans leurs conclusions, les experts proposent un plan de développement pour l'émission et la diffusion, afin que la Télévision sénégalaise ne soit pas taxée de « télévision de capitale ${ }^{71}$ ». S'ils laissent aux autorités le soin de juger si la priorité doit être donnée à l'accroissement de la production ou à l'extension du réseau, ils insistent sur la mise en œuvre des mesures recommandées, notamment l'affectation dès janvier 1972 des assistants techniques, dont le coût annuel est estimé à plus de 16 millions de FCFA ${ }^{72}$. En septembre 1971, l'étude est remise aux autorités sénégalaises, accompagnée de plans et de cartes réalisés par le Service ingénierie - dont du centre de production et de l'emplacement de l'émetteur de Dakar, et des situations des stations de Dakar, Thiès, Kaolack et Ziguinchor.

Pour Guy Bernède, qui doit retourner à son quotidien de la Télévision extérieure, le travail s'arrête là. Quelques jours plus tard cependant, le hasard fait qu'il est contacté par son ami de I'Ocora Radio André Célarié, maintenant à I'ORTF Télé-Lille (devenu France 3 Nord-Pas-deCalais). Ce dernier lui propose de venir gérer le nouveau centre de production lourde de Lambersart, qui alimentera l'antenne nationale de la future Troisième chaîne de l'ORTF, et Guy Bernède, qui ne s'occupe plus beaucoup de technique, accepte d'aller visiter les installations. Le directeur de la Daec Raymond Poussard, prévenu de l'affaire, le convoque à son retour et lui fait une contre-proposition: redresser le centre de Courbevoie. Le service d'envoi de programmes, renommé Agence Inter-TV en décembre 1970, est en effet dans une mauvaise passe. Jacques Anjubault, ancien monteur des premiers JT de Pierre Sabbagh, qui le dirige depuis février 1971, I'a rendu déficitaire ${ }^{73}$.

\footnotetext{
${ }^{68}$ ANF, 28AR(3), télégramme de Guy Bernède à Honorat Ayande, 5 juillet 1971 ; ANF, 28AR(3), devis des Établissements André Debrie pour Dakar, n 17.023, 13 août 1971.

69 Le terme de « tropicalisation » était utilisé pour qualifier le processus d'adaptation des équipements audiovisuels à la situation des pays en développement de climats tropicaux et sahéliens (résistance à la chaleur, à l'humidité et la poussière, accès à la maintenance et aux pièces détachées, etc.).

70 Entretien avec Guy Bernède, 21 et 22 novembre 2019, Boulogne-Billancourt. Nagra est une marque d'enregistreurs sonores portables de haute qualité, utilisés dans le cinéma et la radio à partir des années 1950.

71 ANF, 28AR(3), Télévision au Sénégal. Étude préliminaire, SI/DAEC, août 1971, p. 5.

72 Les sept agents recevront en effet chacun 250000 FCFA de somme forfaitaire mobilier, puis 100000 FCFA de salaire et 75000 FCFA de loyer chaque mois. Dans le même temps, le salaire mensuel des agents sénégalais n'excède pas 90000 FCFA à l'échelon le plus élevé. ANF, 28AR(3), budget télévision 1970-72, n.d.

73 Les archives de la Télévision extérieure de 1970-1971 conservées dans le Fonds Guy Bernède font plus généralement état de nombreux problèmes dans la gestion du centre (sujets non expédiés ou en retard, tirés à l'envers, personnel absent, équipement défaillant, crédits insuffisants, etc.). ANF, 28AR(5).
} 


\section{Guy Bernède, rédacteur en chef de l'Agence Inter-TV}

Guy Bernède accepte la proposition et prend ses fonctions en janvier 1972. Dès le 18 janvier, il s'envole pour une mission circulaire Haute-Volta - Côte d'Ivoire - République populaire du Congo (ex-République du Congo) - Gabon, en vue de faire le point avec les télévisions sur les programmes fournis par Inter-TV et la Télévision extérieure ${ }^{74}$. À chaque étape, il s'entretient avec les mêmes interlocuteurs, selon le même mode opératoire : le ministre de I'Information, les cadres et les assistants de la télévision, les équipes de l'ambassade de France et de la Mac.

Les relations ont changé avec la fin de l'Ocora et, bien que son compte rendu précise que sa mission a été bien accueillie, certaines annotations font au contraire état d'une «vie nerveuse » faite de tensions et de jeux de pouvoirs dans les stations, où les différents groupes d'acteurs (cadres des télévisions, coopérants, réseaux consulaires) cherchent à préserver leur influence ${ }^{75}$. Au sujet de la Radiodiffusion télévision ivoirienne (RTI) par exemple, Guy Bernède mentionne que sa visite a débuté "dans un climat un peu "froid" en raison de la récente visite d'un collaborateur de l'Office » mais que M. de Reynaud, le conseiller technique français du ministre de l'Information M. Bouazo, est intervenu afin que son « enquête sur les programmes puisse se dérouler normalement ${ }^{76}$ ». II mentionne encore que M. Revel, l'assistant technique du chef du Service des programmes Mamadou Ben Soumahoro, ne lui a jamais fourni les documents dont il avait besoin pour son enquête.

Sur le plan matériel à cette période, les situations des télévisions sont très contrastées. En Côte d'Ivoire, la situation est bonne. La RTI diffuse tous les jours pour un volume mensuel de 146 heures et elle va officiellement ouvrir son Studio-école en avril 1972. La capacité de production de la chaîne reste néanmoins limitée, impliquant que peu d'émissions locales sont diffusées (essentiellement les directs) et qu'elle a un fort besoin de programmes extérieurs pour alimenter et étendre son temps d'antenne. Mamadou Ben Soumahoro communique ainsi à Guy Bernède une liste des programmes qu'il souhaite recevoir dans le cadre de l'assistance et comme la RTI dispose maintenant d'un budget pour l'achat de programmes, il joint également une liste de programmes payants ${ }^{77}$.

En Haute-Volta en revanche, Voltavision connaît de graves difficultés financières ${ }^{78}$. Si la station émet maintenant quatre jours par semaine, son volume mensuel d'émissions n'est que de 48 heures environ, trois fois moins que la RTI. Sous-équipée, elle ne dispose que de six agents non qualifiés, deux stagiaires étant alors en formation à l'ORTF. Quelques productions locales sont diffusées, mais des incidents techniques font souvent annuler ces émissions. Du fait de son maigre budget, il est impossible pour Voltavision d'acquérir des programmes à titre

\footnotetext{
74 Outre les actualités et magazines Inter-TV, des « programmes culturels » sont en effet transmis par l'ORTF aux stations africaines via la Télévision extérieure.

75 Sur la «vie nerveuse » de la colonie, voir Mbembe Achille (2006), « La colonie : son petit secret et sa part maudite », Politique africaine, 102, pp. 101-127.

76 ANF, 28AR(2), compte-rendu de la mission de M. Guy Bernède - Haute-Volta, Côte d'Ivoire, Congo-Brazzaville, Gabon, 18 janvier3 février 1972, p. 7.

77 ANF, 28AR(2), compte-rendu de la mission..., rap. cité, pp. 7-8. Ces programmes acquis à titre onéreux comprennent sept films de long métrage, une série de quatre épisodes, un magazine et deux combats de catch par mois. Les programmes gratuits représentent une trentaine d'heures mensuelles et comprennent dramatiques, représentations de théâtre, variétés, émissions de jazz, émissions jeune public, feuilletons, ainsi qu'un nombre important de documentaires (santé, science, tourisme, monde animal).

78 En effet, son budget annuel n'a été que de 2,5 millions de FCFA en 1971. Ibid., p. 3.
} 
onéreux et l'essentiel de sa grille est constitué de programmes étrangers et gratuits. Odette Sanogoh, cheffe du Service des programmes, transmet à Guy Bernède une liste de ceux qu'elle souhaite recevoir (huit heures hebdomadaires). Ce manque de fonds pose un autre problème de taille à la station, celui de la réexpédition des programmes à Paris après leur diffusion. Les colis sont en effet volumineux, généralement autour de cent kilos, et les frais de transport trop élevés ${ }^{79}$. La station ne peut plus payer et au moment où Guy Bernède est à Ouagadougou, elle a un arriéré auprès d'Air Afrique qui menace de bloquer les films. L'aide demandée à l'ORTF est dès lors massive. II s'agit notamment de fournir des programmes, d'assurer leur acheminement et réexpédition et de former du personnel.

Au-delà de ces contrastes, Guy Bernède fait toutefois un même constat. Malgré le fort besoin en programmes extérieurs, les séquences d'actualités Inter-TV sont très peu diffusées par les télévisions, contrairement aux « programmes culturels » de la Télévision extérieure. Les raisons qu'elles avancent sont peu ou prou les mêmes. Le délai d'acheminement est trop long, ce qui est problématique pour de l'actualité, mais il y a aussi des raisons de fond. Les sujets sont inadaptés, trop franco-centrés (même si le terme n'est pas utilisé) et le plus souvent déjà commentés, ce qui laisse peu de latitude aux rédactions pour exploiter ces images comme elles I'entendent dans leurs JT. Mais pour Guy Bernède, cette faible utilisation des séquences est aussi liée à un autre facteur : la concurrence des autres agences et administrations étrangères. En effet, les télévisions africaines reçoivent des programmes de nombreuses ambassades et sont, comme I'ORTF lui-même, abonnées au service français de l'agence britannique Visnews (Reuters-BBC) afin d'alimenter leurs JT en actualités internationales ${ }^{80}$. C'est le cas de la RTI, dont Guy Bernède dit que la concurrence y est rude ${ }^{81}$. II y a aussi la banque de programmes ouestallemande Transtel, dont toutes les télévisions sont partenaires, et dans un contexte où les États africains installent leurs premières stations terriennes, il suggère à sa hiérarchie de se positionner :

Après I'ORTF le principal fournisseur de programmes à ces stations est sans aucun doute Transtel (feuilletons, séries, variétés, etc.). Les États-Unis offrent des shows [...], des émissions scientifiques et documentaires [...]. L'Italie offre un "panorama italien », assez mal réalisé paraît-il. Le Congo reçoit en plus des productions de l'Europe de l'Est et de la Chine populaire. À Brazzaville, j'ai appris que la Télévision de Kinshasa avait déjà retransmis en direct des émissions en provenance d'Allemagne, grâce à l'utilisation du satellite Intelsat et avait annoncé, pour l'été prochain, la diffusion des Jeux olympiques en direct. Madagascar, le Gabon et la Côte d'Ivoire auront bientôt leur station de réception. II faudrait étudier dès maintenant les possibilités offertes par ce nouveau mode de transmission et, bien entendu, les moyens nécessaires pour éviter d'être complètement supplanté par les Allemands ou les Américains. ${ }^{82}$

\footnotetext{
79 Ibid. En 1971, ils se sont élevés à 460197 FCFA, grevant d'environ $20 \%$ le budget de la télévision.

${ }^{80}$ Bien que cette information n'ait pu être vérifiée, Guy Bernède explique que Visnews, créée en 1957, avait offert au moment des indépendances un service gratuit durant trois jours aux États africains.

${ }^{81} \mathrm{ANF}, 28 \mathrm{AR}(2)$, compte-rendu de la mission..., rap. cité, p. 8.

$82 \mathrm{Ibid}$, p. 2. Les satellites de télécommunications sont alors le monopole de la société privée états-unienne Comsat (réseau Intelsat), créée en 1962.
} 


\section{Les premières transmissions satellitaires et le démarrage de la Télévision sénégalaise}

Cette mission change la donne. Fort de ses enseignements, Guy Bernède décide de créer un "Magazine africain » afin de reconquérir les antennes ${ }^{83}$. La production d'Inter-TV se développe et il bénéficie d'une certaine latitude dans son travail, car le financement est directement assuré par les ministères des Affaires étrangères et de la Coopération. En contact permanent avec la Direction de la coopération culturelle et technique et avec l'appui de son interlocutrice Geneviève Dindin, il en profite pour augmenter le volume de programmes : "Je connaissais les besoins des télévisions, alors si au lieu de leur envoyer cinq heures [...] je pouvais envoyer six heures, j'étais très content pour eux et eux aussi ${ }^{84}$ ».

Sur le plan matériel, il doit également trouver d'autres moyens d'acheminer les programmes et un événement lui donne cette opportunité dès juillet 1972. En effet, tous les après-midis à cette période, les membres de I'Union européenne de radio-télévision (UER) s'échangent des actualités par faisceaux (Eurovision News). Israël et la Jordanie, qui viennent de s'équiper de stations terriennes, demandent à recevoir le service par satellite. L'UER accepte et l'ORTF est retenu pour assurer la sélection-transmission, avec comme façonnier le Service des relations internationales de la Télévision extérieure ${ }^{85}$. Le service, qui démarre le 27 juillet, requiert une certaine technicité : "À l'époque c'était les magnétos deux pouces, des grosses machines énormes. Ils faisaient une sélection [...] et ils renvoyaient ça [...] dans une tranche satellite de dix minutes. Ça coûtait cher et c'était difficile à obtenir le satellite dix minutes ${ }^{86}{ }^{\prime}$.

Lorsqu'il apprend cela, Guy Bernède décide de reprendre le dossier sénégalais. Sandor Polgar et lui avaient préconisé le démarrage des émissions pour la fête nationale le 4 avril 1972, mais le projet piétine et celui-ci n'a pas eu lieu. II sait toutefois qu'une station terrienne a été implantée au premier semestre à Gandoul, à 70 kilomètres de Dakar, avec un nouvel émetteur de 100 Watts couvrant la presqu'île du Cap Vert (Dakar et sa périphérie). Le faisceau hertzien entre Gandoul et la Télévision, située sur la presqu'île, n'est pas encore installé, mais il décide de tenter quelque chose pour les Jeux olympiques (JO) de Munich qui démarreront en août. Durant sa mission, Guy Bernède a constaté que les télévisions les attendent avec impatience et que certaines, comme à Kinshasa, sont déjà préparées. Ancien patron des sports de RL, il reste par ailleurs journaliste sportif de cœur et a une expérience des JO, qu'il couvrira à Munich et a déjà couverts en 1960 à Rome pour RL et en 1964 à Tokyo pour l'Ocora. Ayant appris qu'une station a aussi été installée en Martinique pour le téléphone, il convainc Raymond Poussard de les desservir ensemble :

On avait réussi à obtenir un tarif préférentiel parce qu'il y avait un département français, [...] le tarif domestique a pu être appliqué [...]. [Mais] le faisceau descendant était à la charge de I'utilisateur, [...] parce que quand on ne paye pas on n'apprécie pas. J'avais connu ça avec les

\footnotetext{
${ }^{83}$ Ce magazine est constitué de sujets liés à l'Afrique tournés en France. En effet, au moment où Guy Bernède prend ses fonctions, Inter-TV compte une centaine de télévisions partenaires sur les cinq continents et des magazines ont été créés en plusieurs langues (le Magazine littéraire, le magazine féminin « Pour vous Madame » et le Magazine scientifique), mais aucun pour les téléspectateurs africains.

84 Entretien avec Guy Bernède, 21 et 22 novembre 2019, Boulogne-Billancourt.

85 Ce service, basé à I'ORTF rue Cognacq-Jay, s'occupait des rapports avec I'UER et les télévisions étrangères.

86 Entretien avec Guy Bernède, 21 et 22 novembre 2019, Boulogne-Billancourt.
} 
envois que faisaient en radio les Américains après la [Seconde] Guerre [mondiale], ils nous envoyaient des disques à RL qu'on n'écoutait même pas, parce que c'était gratuit. ${ }^{87}$

Guy Bernède prépare alors un montage. Le signal partira de Munich vers le site de l'ORTF rue Cognacq-Jay (Paris), d'où il sera redirigé par câble vers Pleumeur-Bodou, première station émettrice installée dans les Côtes-d'Armor. II se met en relation avec les Postes et télécommunications pour louer le satellite vingt minutes, juste après les dix minutes Israël/Jordanie. Outre le direct de Munich, les transmissions incluront des actualités de l'ORTF, une sélection qui sera ajoutée lors du transit du signal par Cognacq-Jay.

Tout est prêt mais Guy Bernède réalise qu'il y a un problème de taille : la société ouestallemande Transtel détient les droits de retransmission exclusifs pour l'Afrique. II connaît bien leurs représentants, qu'il rencontre depuis 1963 aux assemblées générales de l'Urtna, et se rend à Cologne où il négocie avec le Dr. Krause-Brewer, président de Transtel, afin de pouvoir envoyer des "émissions spéciales Afrique » et un résumé quotidien francophone durant les JO. Transtel n'a pas de journaliste francophone et c'est ce qui va permettre de trouver un accord. En effet, le secrétaire de rédaction d'Inter-TV Richard Hartzer, Alsacien d'origine, est bilingue français-allemand et il propose de le mettre à leur disposition. Le Dr. Krause-Brewer accepte et Richard Hartzer rejoint l'équipe en partance pour Munich. Tous les soirs, il se rend chez Transtel pour produire le résumé francophone et en échange, Guy Bernède peut reprendre et réaliser les images qu'il souhaite. Cette coproduction Transtel/Inter-TV, unique en son genre, est envoyée par avion aux télévisions africaines via Paris.

La Télévision sénégalaise démarre le 24 août 1972, deux jours avant le début des JO et le premier direct satellite. Ces émissions expérimentales alternent films réalisés par le Service cinéma du ministère de I'Information sénégalais, que dirige le cinéaste Paulin Soumanou Vieyra, et mire de la Télévision sénégalaise. Des films sont également envoyés de Paris et un assistant est dépêché à Dakar par Guy Bernède pour assurer leur diffusion :

[II] est parti huit jours avant simplement. C'est comme ça que ça a démarré, la télévision de Dakar. [...] [II] a appliqué la grille de programmes que j'avais préparée, donc je n'ai pas été vraiment au démarrage [...]. J'ai fait les études j'ai fait tout ça mais je n'ai pas été présent, [...] alors que j'aurais dû être le patron de la station. ${ }^{88}$

\footnotetext{
87 Ibid. Le tarif est alors de 310000 Francs pour le Sénégal et de 140000 Francs pour la Martinique, les frais de descente de la Martinique étant pris en charge par I'ORTF.

88 Entretien avec Guy Bernède, 21 et 22 novembre 2019, Boulogne-Billancourt.
} 
Illustration $n^{\circ} 3$ : Grille de démarrage de la télévision sénégalaise ${ }^{89}$

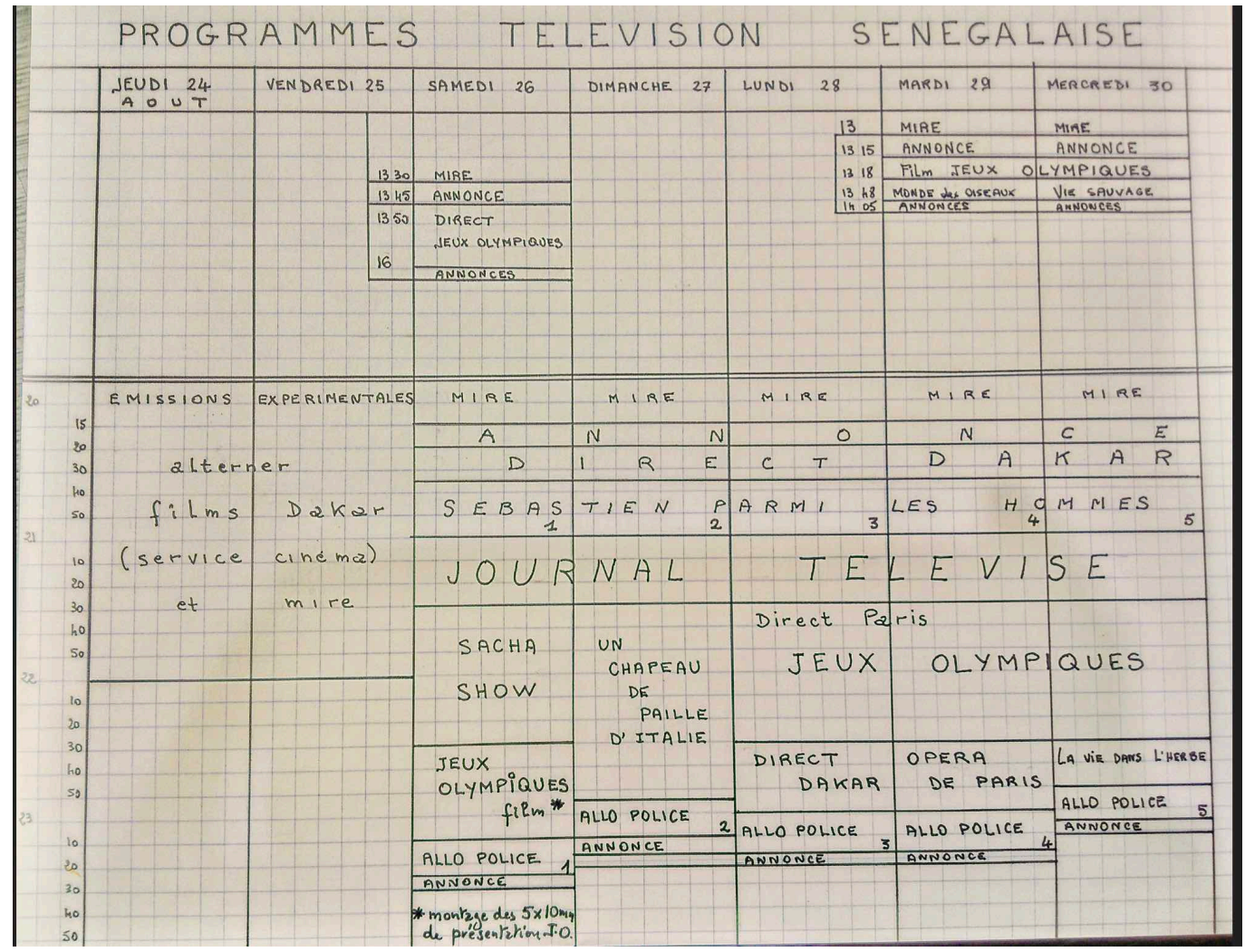

Les transmissions satellitaires sont réussies et, de retour des JO, Guy Bernède monte un petit service d'actualités. En effet, n'ayant pu louer le satellite pour une durée inférieure à un mois, il a préparé une grille mensuelle pour la Télévision sénégalaise et va continuer de fournir des programmes quelques jours. Fin octobre 1972, lors du Sommet européen de Paris, il convainc Raymond Poussard de reprendre le service :

On a continué nos fameuses vingt minutes [...] et puis au fur et à mesure, il y avait des créations de nouvelles stations terriennes. J'avais de nouveaux clients, et ça s'est répandu assez rapidement. [...] On a demandé aux télévisions africaines ce qu'elles en pensaient, elles ont dit que c'était bien, si bien qu'on [...] a été obligés de continuer ${ }^{90}$.

L'ORTF Martinique, qui a permis à Inter-TV de mettre sur pied la transmission des JO, souhaite continuer de recevoir ces sélections quotidiennes (monitorings). Leur contenu est ainsi modifié afin d'intégrer cinq minutes destinées aux Dom-Tom, la Martinique effectuant une copie qu'elle expédie par avion en Guadeloupe. Les quinze autres restent destinées à l'Afrique, soit quatre sujets de trois-quatre minutes environ. La Télévision sénégalaise, qui poursuit ses émissions quotidiennes à Dakar, reprend le service. Le gouvernement, qui souhaite étendre la diffusion au reste du pays, passe par ailleurs une commande de matériel à Thomson-

\footnotetext{
${ }^{89}$ ANF, 28AR/3.

90 Entretien avec Guy Bernède, 21 et 22 novembre 2019, Boulogne-Billancourt.
} 
CSF ${ }^{91}$. D'autres États africains ouvrent progressivement des stations terriennes et s'abonnent au service. C'est le cas du Zaïre (ex-RDC) le 24 octobre 1972, puis de la Côte d'Ivoire le $1^{\text {er }}$ novembre 1972. Guy Bernède devient de fait responsable de la mise en place du relais des programmes français par satellite à la Daec.

Inter-TV continue aussi d'expédier des programmes par le fret ${ }^{92}$. Ces envois sont adressés aux ambassades de France afin de réduire les frais de douane, mais pour l'Afrique le circuit reste différent: "Les Africains c'était à part, on envoyait directement aux télévisions. [...] On continuait comme on faisait du temps de l'Ocora93 ». À cette période, les volumes de programmes en circulation sont tels qu'un bureau permanent de DHL est installé à Courbevoie. Néanmoins, le satellite modifie en substance le travail d'Inter-TV :

Quand on reprenait un sujet de l'ORTF, c'était un sujet qui datait déjà peut-être de 24 heures. [...] Le temps qu'on le monte, qu'on l'envoie (s'il y avait un avion tout de suite, parce qu'il n'y avait pas des avions tous les jours), ils recevaient avec trois-quatre jours au mieux de retard. Ils le diffusaient, alors je disais «C'est pas de l'actualité » [...]. J'étais toujours à l'affut des nouvelles techniques, alors quand il y a eu ces transmissions par satellite j'ai dit « C'est ça ! » [...] Je suis revenu finalement à Courbevoie au bon moment, [...] [pour les] JO. Tout ça s'est bien enchaîné, et j'ai pu lancer ça [les monitorings]. Et j'ai été aidé par Poussard, comme directeur, parce qu'après j'ai eu des directeurs qui ne m'ont pas aidé. ${ }^{94}$

En effet, la situation d'Inter-TV va vite se dégrader. Fin 1972, Raymond Poussard est remplacé par Claude Contamine, qui multiplie les coupes budgétaires. Avec le nouveau directeur de la Télévision extérieure René Han, il prend la décision de vendre le centre fin 1973 et Inter-TV est rapatrié dans des locaux près de Cognacq-Jay. Amputé de ses capacités de production, le service se démantèle. La cinémathèque, qui conservait les programmes produits depuis 1962, est également envoyée à la destruction sur ordre du chef de centre Bernard Avran, sans que Guy Bernède ait été prévenu.

\section{De la Daec à FR3 Dom-Tom (1974-1975)}

En décembre 1974, la dissolution du service s'achève avec le démantèlement de l'ORTF. En janvier 1975, sept nouvelles sociétés sont créées : Radio France, Télévision française 1 (TF1), Antenne 2 (A2), France Régions 3 (FR3), la Société française de production (SFP), TéléDiffusion de France (TDF) et l'Institut national de l'audiovisuel (Ina) ${ }^{95}$. S'il est un temps envisagé d'en créer

\footnotetext{
91 Celle-ci comprend un car de reportage avec faisceau hertzien mobile, deux télécinémas 16 mm, des caméras, ainsi qu'un émetteur de $10 \mathrm{~kW}$ avec antenne omnidirective à grand gain. ANF, 28AR(3), Thomson-CSF au Sénégal, Nouvelles Thomson-CSF, Division Radiodiffusion Télévision, F/029, décembre 1972.

92 En 1972, 894 heures d'actualités et 223 heures de magazines sont ainsi exportées dans le monde. Voir ANF, 28AR(5), bilan InterTV 1972, n.d.

93 Entretien avec Guy Bernède, 21 et 22 novembre 2019, Boulogne-Billancourt.

94 Ibid.

95 La SFP hérite des activités de production de I'ORTF, TDF de la gestion de ses émetteurs, et I'Ina de ses archives audiovisuelles et de la formation. TF1, A2 et FR3 elles, succèdent aux première, deuxième et troisième chaînes de l'ORTF (FR3 récupérant ses anciennes antennes régionales). Radio France enfin, hérite des quatre chaînes radio nationales : France Musique, France Inter, France Culture et France Inter Paris (Fip). Elle hérite également des enregistrements ethnomusicologiques réalisés par l'Ocora dans les années 1960 et récupérés par la Daec en 1969, «Ocora » devenant alors un label discographique de Radio France, jusqu'à aujourd'hui.
} 
une huitième chargée de l'international, les activités de coopération que gérait la Daec sont finalement réparties entre elles ainsi que son personnel, qui continue de travailler ensemble ${ }^{96}$.

Dans cette restructuration, l'envoi des « programmes culturels » aux télévisions africaines est attribué à I'Ina. Celui des monitorings, en revanche, se retrouve dévolu à la Direction de FR3 chargée de l'Outre-mer : FR3 Dom-Tom. À cette occasion, le principe est inversé, puisque ce sont des séquences d'actualités sélectionnées pour les Dom-Tom qui sont envoyées aux télévisions africaines. Ces dernières vivent d'autant plus mal cette situation que celle-ci ramène symboliquement, quinze ans après l'indépendance, les États africains dans le giron de la France ultramarine ${ }^{97}$. Guy Bernède, qui a été nommé administrateur des journalistes d'Outre-mer, reçoit de nombreuses plaintes :

À FR3 grosse bêtise, les Dom-Tom ! [...] On m'a dit « C'est les Dom-Tom qui vont prendre les monitorings ». Si bien que quand les Africains ont appris ça ils n'étaient pas contents, j'ai reçu des coups de fil. [...] Et puis quand on les a rencontrés à Alger, je me souviens que les directeurs sont venus me dire « On est indépendants depuis! Quand même ! »88

De retour d'Alger, il souligne ce problème dans un rapport qui ne fait l'objet d'aucune suite. Comme il le commente: «II n'était plus possible de changer quoi que ce soit, tant pis pour la fierté africaine ${ }^{99} »$. II explique avoir, lui aussi, mal vécu cet état de fait :

Les Africains auraient dit « C'est du colonialisme » etc. bon, on supprime, on en profite [pour faire des économies]. Mais ce n'était pas ça du tout, on avait d'excellents rapports. J'étais peut-être un petit peu responsable de ça, parce que [...] je connaissais bien la mentalité des différentes stations. Et [...] quand j'étais en Afrique j'étais Africain, je défendais le point de vue africain. Bon, tout en sachant qu'il y avait des problèmes en France et tout ça. ${ }^{100}$

De fait, Guy Bernède va multiplier les oppositions avec sa hiérarchie, qu'il juge afrophobe ${ }^{101}$. Dans un contexte où deux innovations, les vidéocassettes et la vidéo couleur, viennent impacter la coopération télévisuelle avec l'Afrique, il maintient néanmoins le contact avec les télévisions africaines et réussit à remonter un service de monitoring avec un "Spécial Afrique », qui perdure jusqu'à la prochaine restructuration ${ }^{102}$.

\footnotetext{
96 Louis Ménard par exemple, est affecté à TDF, tandis que Guy Violante et Jacques Conia sont affectés à I'Ina.

97 Celle-ci perdure près de huit ans, puisqu'il faut attendre 1982 et la création de Radio France d'Outre-mer (RFO), qui comprend une rédaction Dom-Tom et une Agence internationale d'images de télévision (AITV) dont Guy Bernède est rédacteur en chef, pour que l'Afrique soit à nouveau dissociée des Dom-Tom.

98 Entretien avec Guy Bernède, 21 et 22 novembre 2019, Boulogne-Billancourt. En janvier 1975, Guy Bernède s'est en effet rendu à Alger pour l'assemblée générale de l'Urtna.

99 Ibid.

100 lbid.

101 II raconte notamment que René Han, devenu directeur de FR3 Dom-Tom, lui aurait interdit de couvrir le décès du président Gabriel Ramanantsoa à Paris en mai 1979, au titre qu'il «n'aimait pas les Malgaches ». En complicité avec l'équipe de la Télévision malgache, il tourne tout de même des images et réussit à les faire partir dans l'avion ramenant le corps à Tananarive, où elles sont finalement diffusées.

102 Les vidéocassettes envoyées par la coopération notamment, sont souvent utilisées par les télévisions africaines pour enregistrer des contenus endogènes et pallier le manque de supports vierges, une stratégie rendue possible par la nouvelle technologie magnétique, jusqu'alors impossible à mettre en œuvre avec le film. Voir Losch Flora (2020), "Preserving Public Broadcasting Archives in the Digital Era: Circulatory Stories and Technologies, the Digital Turn, and the Return of the Past in West Africa ", History in Africa, 47, pp. 219-241.
} 
Recruté comme pigiste par l'Ocora pour participer au démarrage de Télé Congo en novembre 1962, Guy Bernède contribua finalement au développement de l'Agence Télévision, dont il devint directeur adjoint dès janvier 1963 après le démarrage manqué de la Télévision sénégalaise. Contraint de quitter le centre de Courbevoie pour rejoindre la Daec après la dissolution de l'Ocora en juillet 1969, il le regagna en janvier 1972 en tant que rédacteur de chef de la nouvelle Agence d'envoi de programmes Inter-TV. Dans le cadre de cette fonction, il initia les premières transmissions satellitaires vers l'Afrique pour assurer le démarrage de la Télévision sénégalaise et devint responsable du relais des programmes français par satellite à la Daec. Affecté à la nouvelle FR3 Dom-Tom en janvier 1975 après le démantèlement de l'ORTF, il fut témoin du revirement de la coopération télévisuelle avec l'Afrique francophone et remonta, contre sa hiérarchie, le "Spécial Afrique », finalement repris par les télévisions africaines jusqu'à la fin des années 1980.

Ainsi, ces différentes étapes de la carrière de Guy Bernède mettent en évidence les recompositions de la coopération télévisuelle entre la France et ses anciennes colonies d'Afrique entre 1962 et 1975, et en révèlent les continuités et les ruptures. Plus généralement, cet itinéraire professionnel nous permet d'observer que la France, après avoir démantelé son empire africain, a cherché à maintenir son influence sur le continent en investissant son nouvel espace télévisuel avec des programmes et des technologies, se livrant à une compétition avec d'autres États. En conséquence, l'histoire des télévisions d'Afrique francophone et l'histoire de la coopération française avec l'Afrique ont été circulatoires et entremêlées après les indépendances de 1960.

Parce qu'il a agi dans ce contexte politique spécifique, Guy Bernède a de ce fait incarné une certaine façon de faire la coopération franco-africaine, basée sur des relations privilégiées et un sentiment d'intimité caractéristiques des années du Secrétariat général des Affaires africaines et malgaches ${ }^{103}$. Comme il le souligne:

Quand ils avaient un problème ils n'hésitaient pas à m'appeler, [...] parce que quand j'étais ici j'étais le représentant africain. [...] Bon en plus, j'avais été un peu le père de ces télévisions, les premières Brazzaville Libreville tout ça, alors on faisait appel à moi. [...] Mais moi, je restais toujours à ma place ${ }^{104}$.

Dans le même temps, parce que Guy Bernède n'avait pas de « carrière africaine » derrière lui, son itinéraire nuance l'analyse de la réaffectation des anciens agents coloniaux dans la coopération ${ }^{105}$. Le récit de ce coopérant, qui demande encore à être confronté aux archives africaines notamment congolaises et sénégalaises et aux autres archives françaises, nous montre enfin que l'histoire des télévisions d'Afrique et l'histoire de la coopération française

\footnotetext{
103 Voir Bat J.-P., Le Syndrome Foccart..., op. cit., p. 727.

104 Entretien avec Guy Bernède, 21 et 22 novembre 2019, Boulogne-Billancourt.

105 Voir notamment Tiquet Romain (2013), « Un policier français dans l'Empire. Pierre Lefuel, dernier directeur de la Sûreté voltaïque (1959-1960) et pionnier du Service de coopération technique internationale de police (SCTIP) ", Histoire, économie \& société, 4, pp. 49-58; Dimier Véronique (2014), The Invention of a European Development Aid Bureaucracy. Recycling Empire, Basingstoke, Palgrave Macmillan.
} 
avec l'Afrique ont été enchâssées dans une autre histoire, celle des acteurs, devant être nécessairement prise en compte afin de saisir cette chronologie post-coloniale dans sa complexité.

Flora Losch

Centre Alexandre-Koyré, EHESS (France)

\section{Bibliographie}

BAT Jean-Pierre (2012), Le Syndrome Foccart. La politique française en Afrique, de 1959 à nos jours, Paris, Gallimard.

Векомво Manga (1966), "Brazzaville à I'heure de la télévision congolaise », Revue française de sociologie, 7(2), pp. 188-200.

BOURDON Jérôme (2014 [1990]), Histoire de la télévision sous de Gaulle, Paris, Presses des Mines.

Colin Roland, PERROT Thomas et SMITH Étienne (2010), " "Alors, tu ne m'embrasses plus Léopold ?" Mamadou Dia et Léopold S. Senghor », Afrique contemporaine, 233, pp. 111-132.

DAmome Étienne L. (2012), « Vers un réseau Outre-mer », in M. KALTENECKER et K. Le BAlL (dir.), Pierre Schaeffer. Les constructions impatientes, Paris, CNRS, pp. 164-177.

DIMIER Véronique (2014), The Invention of a European Development Aid Bureaucracy. Recycling Empire, Basingstoke, Palgrave Macmillan.

Dindane Ousmane (2016), De l'arrière du décor. Une odyssée au cœur de la Télévision ivoirienne, Abidjan, L'Encre Bleue.

Dı̈ Tidiane (2009), Histoire de la télévision en Afrique noire francophone, des origines à nos jours, Paris, Karthala.

GOERG Odile et RAISON-JOURDE Françoise (dir.) (2012), « Les coopérants français en Afrique : portrait de groupe (années 1950-1990) », Cahiers Afrique, 28.

Goerg Odile et De SUREMAIN Marie-Albane (dir.) (2014), "Coopérants et coopération en Afrique: circulations d'acteurs et recompositions culturelles (des années 1950 à nos jours) ", Outre-Mers. Revue d'histoire, 384-385.

Grandmougin Jean (1967), Lettre ouverte au Ministre de I'Information, Paris, Albin Michel.

KIRIAKOU Héloïse (2019), « Ébranler la gérontocratie au pouvoir : quand les cadets font la révolution au Congo-Brazzaville", in Y. DENECHERE (dir.), Enjeux postcoloniaux de l'enfance et de la jeunesse. Espace francophone (1945-1980), Bruxelles, Peter Lang, pp. 192-202.

LAUZANNE Bernard (1992), "L'affaire Grandmougin : comment fut contraint au silence "I'homme le plus écouté de France" », Cahiers d'histoire de la radiodiffusion, 35, pp. 54-60bis.

LOSCH Flora (2020), «Preserving Public Broadcasting Archives in the Digital Era: Circulatory Stories and Technologies, the Digital Turn, and the Return of the Past in West Africa », History in Africa, 47, pp. 219241. 
Mbembe Achille (2006), "La colonie: son petit secret et sa part maudite », Politique africaine, 102, pp. 101-127.

PeRALDI Michel (dir.) (2016), «Mobilités et migrations européennes en (post) colonies », Cahiers d'études africaines, 221-222.

NicolAS Claire (2019), " De I'histoire comparée à la démarche multi-située: sur les traces des cadres sportifs africains », Espaces et Sociétés, 178, pp. 55-72.

QUASHIE Hélène (2016), « Débuter sa carrière professionnelle en Afrique. L'idéal d'insertion sociale des volontaires français à Dakar et Antananarivo (Sénégal, Madagascar) ", Cahiers d'études africaines, 221 222, pp. 53-80.

Schaeffer Pierre (1978), Les Antennes de Jéricho, Paris, Stock.

TIQUET Romain (2013), « Un policier français dans l'Empire. Pierre Lefuel, dernier directeur de la Sûreté voltaïque (1959-1960) et pionnier du Service de coopération technique internationale de police (SCTIP) », Histoire, économie \& société, 4, pp. 49-58.

TUDESQ André-Jean (1992), L'Afrique noire et ses télévisions, Paris, Ina/Anthropos.

VeYne Paul (1971), Comment on écrit l'histoire, Paris, Seuil. 